Check for updates

Cite this: RSC Adv., 2017, 7, 42172

Received 19th July 2017

Accepted 23rd August 2017

DOI: $10.1039 / \mathrm{c} 7 \mathrm{ra07956g}$

rsc.li/rsc-advances

\section{Biofilm evolution and viability during in situ preparation of a graphene/exoelectrogen composite biofilm electrode for a high- performance microbial fuel cell}

\author{
Feng Deng, ${ }^{a}$ Jian Sun, (D) ${ }^{b}$ Yongyou Hu, (DD *a Junfeng Chen, (D) a Sizhe Li, ${ }^{\text {a }}$ Jie Chen ${ }^{a}$ \\ and Yaping Zhang ${ }^{\mathrm{b}}$
}

\begin{abstract}
To understand how microbial reduction of graphene oxide ( $\mathrm{mrGO}$ ) influenced the biofilm during the in situ preparation of a graphene/exoelectrogen composite biofilm anode in a microbial fuel cell (MFC), an in situ mrGO-modified anode was fabricated, and the evolution and viability of the anodic biofilm were investigated by sampling the biofilm at different stages of the MFC operation. The total protein, thickness and viability of the anodic biofilm in $\mathrm{mrGO}$-added MFCs were generally lower than that in $\mathrm{mrGO}$-free MFC at the first electricity production cycle (EPC) due to the antibacterial activity of the GO flake. However, the three indicators increased dramatically and were much higher in mrGO-added MFC than in mrGO-free MFC after three EPCs, derived from enhanced bacterial adhesion and accelerated biofilm recovery in the presence of $\mathrm{mrGO}$ due to its high specific area for increased biomass attachment and high conductivity for enhanced electron transfer between bacteria and anode. The electricity generation performance of the MFC was found to follow the same trend as biofilm evolution. The power density of the mrGO-modified MFC increased sharply after five electricity production cycles, reaching a maximum value of $1140.63 \mathrm{~mW} \mathrm{~m}^{-2}$, which is $65.20 \%$ higher than the blank control. This study gives an insight into the interaction between the exoelectrogens and their induced GO reduction which contribute to the understanding of the essence of the process for high-performance MFC application.
\end{abstract}

\section{Introduction}

Microbial fuel cells (MFCs) are devices that can use organic or inorganic substances through microbial metabolism to produce clean and renewable energy, ${ }^{1-3}$ however, the relatively low power density and poor energy conversion efficiency of MFCs limit their practical applications. Microorganisms grown as a biofilm on the anode surface are crucial for anode performance because they directly correlate with substrate metabolism and electricity harvesting from the substrate using the anode as an electron acceptor. ${ }^{4-6}$ Numerous efforts have been made to optimize the materials and structure of MFC anodes so as to facilitate bacterial adhesion and extracellular electron transfer (EET) from bacteria to anode. Among these efforts, nanostructured

\footnotetext{
${ }^{a}$ Ministry of Education Key Laboratory of Pollution Control and Ecological Remediation for Industrial Agglomeration Area, Department of Environmental Science and Engineering, College of Environmental Science and Engineering, South China University of Technology, Guangzhou, 510006, China. E-mail: ppyyhu@scut. edu.cn; Fax: +8620 39380508; Tel: +86 2039380506

${ }^{b}$ School of Environmental Science and Engineering, Institute of Environmental Health and Pollution Control, Guangdong University of Technology, Guangzhou 510006, China
}

materials have been proved to significantly improve MFC output power density.

Graphene, which is an emerging two-dimensional structure, possesses many analogous characteristics, such as excellent electrical and thermal conductivity, ${ }^{7-9}$ a high specific surface area $\left(2675 \mathrm{~m}^{2} \mathrm{~g}^{-1}\right)^{10}$ and a one-atom-thick $2 \mathrm{D}$ structure, ${ }^{8}$ which is broadly utilized in the area of electrochemistry. ${ }^{11-21}$ To date, the known method for fabrication of graphene includes micro mechanical exfoliation, ${ }^{22}$ chemical vapor deposition, ${ }^{23} \mathrm{SiC}$ surface extension ${ }^{24,25}$ and reduction of graphene oxide. However, unfavorable conditions, including toxic material and high temperatures, are involved in the routes mentioned above. Thus, graphene has some negative characteristics, such as hydrophobicity, poor bio-compatibility and potential toxicity. ${ }^{26}$ In recent years, researchers have discovered a greener method of reducing graphene oxide, and some studies have shown that graphene oxide could be reduced by bacteria such as Escherichia coli ${ }^{27}$ which possess an excellent extracellular electron transfer ability. Huang proved that graphene oxide can promote extracellular electron transfer in Shewanella. ${ }^{28}$ Based on these studies, microbial reduction of graphene oxide in the MFC system was investigated by Yuan et al..$^{29,30}$ They demonstrated that microbially reduced graphene scaffolds could facilitate 
extracellular electron transfer in the anode and enhance oxygen reduction in the biocathode.

The conclusions of the previous studies were similar in that the mrGO could be formed in situ on the surface of the anode, which notably enhanced the MFC performance. In order to deeply understand the mechanism of the improvement in electricity generation, it was necessary to investigate the impact of the mrGO on the biofilms during long-term operation. Anodic biofilms could be damaged by both the GO and the mrGO flakes at the earliest stage of the GO dispersion addition because of their sharp edges, ${ }^{31}$ which significantly influence the viability and quantity, and thus, further research is needed. For long-term operation, the fall off of biofilm, the hydraulic actions of media replacement and the maintenance of a high concentration of mrGO flakes have a significant impact on the biofilm evolution and the stability of power density in the MFC system, which also need further discussion.

This paper reports measurements of the in situ formation of the mrGO on the surface of the anode and discovers the patterns of viability and quantity of the anodic biofilms, with the mrGO modifying different test timing points. It is finally understood how the mrGO influences the anodic biofilms, whereby it helps the biofilm evolution and maintains stability during long-term operation. The measurement and falling off of the anodic biofilm after media replacement was considered an indicator of the biofilm recovery analysis. Another indicator was the variation of the biofilm thickness and viability between the neighboring electricity production cycles.

\section{Material and methods}

\subsection{Synthesis of GO}

GO was prepared from graphite powder (Sinopharm Chemical Reagent Co., Ltd., China) using modified hummers, and it was used as supplied. GO was treated by sonication and centrifugation to synthesize graphene oxide. Sonication was conducted in a continuous mode at a delivered power of $150 \mathrm{~W}$ for 2 hours. Next, centrifugation was performed at $3000 \mathrm{rpm}$ for $30 \mathrm{~min}$, and the supernatant was a dispersion of graphene oxide. ${ }^{32,33}$

\subsection{MFC setup}

The MFC consisted of an anode and cathode placed on opposite sides in a plastic cylindrical chamber, which was $2.1 \mathrm{~cm}$ long and $5 \mathrm{~cm}$ in diameter, with a net volume of $40 \mathrm{~mL}$. The anode electrodes were made of graphite felt, and the air-cathode was made of carbon paper $(5 \mathrm{~cm} \times 5 \mathrm{~cm})$ containing $0.5 \mathrm{mg} \mathrm{cm}{ }^{-2}$ of a Pt catalyst. The cation exchange membrane and the PTFE waterproof layers were set together to isolate the substance of the anode and the carbon felt electrode.

Prior to use, both the carbon felt and the carbon paper were cleaned by soaking in acetone overnight. Each single MFC was inoculated with anaerobic-activated sludge present in the biochemical process of the Liede municipal wastewater treatment plant (Guangzhou, China).

\subsection{MFC operation}

A preliminary experiment was performed to study the in situ formation of the mrGO-modified anode, which had a base material of graphite felt. In the experiment, the GO dispersion was added before the start of the MFC.

To study the biofilm evolution and viability on the mrGOmodified anode, parallel experiments were carried out with three MFCs with different startup and operating procedures. We applied two mrGO-modified anodes with different modes of GO dispersion addition: the GO dispersion was only added at the beginning of the first electricity production cycle (MFC-B), and the GO dispersion was added before every electricity production cycle (MFC-C). Both MFCs were systematically compared with the mrGO-free MFC-A, which was used as a blank control. For the formal experiment, dispersion of the GO $\left(1 \mathrm{mg} \mathrm{mL}^{-1}\right)$ was added into the anode chamber of the two different MFCs, where the GO was reduced by anodic microbes and the mrGO was formed in situ on the surface of anode.

When the voltage dropped below $20 \mathrm{mV}$, the medium and water of each reactor were periodically refreshed.

The anodic medium contained $0.5 \mathrm{~g} \mathrm{~L}^{-1}$ sodium acetate, a phosphate-buffered solution (PBS, $50 \mathrm{mM}$ ), a mineral solution $\left(12.5 \mathrm{~mL} \mathrm{~L}^{-1}\right)$ and a vitamin solution $\left(12.5 \mathrm{~mL} \mathrm{~L}^{-1}\right){ }^{34}$

Experiments were conducted in duplicate at room temperature $\left(30 \pm 1{ }^{\circ} \mathrm{C}\right)$, and the average values with the standard deviations were reported. In each MFC, the circuit was connected between anode and cathode by titanium wires with an external resistance of $500 \Omega$.

\subsection{Analysis and calculations}

2.4.1. Qualitative and morphology of mrGO-modified anode. Slices of anodes in different MFCs were removed in the first, third and fifth electricity production cycles, after which they were tested by field emission scanning electron microscope (FESEM, Nova Nano SEM 430, FEI, USA), X-ray diffraction and Raman spectroscopy (Horiba Jobin Yvon, France).

2.4.2. Electrochemical test. The voltage difference between the anode and cathode $(V)$ of each MFC was recorded every 10 min with a data acquisition system (Model 2700, Keithley Instruments, USA). Polarization data were measured by varying the external resistor $\left(R_{\mathrm{ex}}\right)$ over a range of 50 to 1000 at the open circuit. Anode potentials of the MFC were measured against a $\mathrm{Ag} / \mathrm{AgCl}$ electrode (saturated with $\mathrm{KCl}$ ), which was set in the anode chamber. Electrochemical impedance spectroscopy (EIS) was measured using an electrochemical workstation (Model 2273, Princeton Applied Research). For EIS, a three-electrode system was tested by an alternating current signal with amplitude of $10 \mathrm{mV}$, and the frequencies ranged from $10 \mathrm{kHz}$ to $5 \mathrm{mHz}$.

2.4.3. Biofilm analysis. For confocal scanning laser microscopy (CSLM), the anodes were cut into small pieces from devices, washed with a nutrient-free phosphate buffer solution, fluorescently stained using a FilmTracer ${ }^{\mathrm{TM}}$ LIVE/DEAD® Biofilm Viability Kit (Molecular Probes) and examined with a Zeiss LSM 700 inverted microscope. Three random representative images were taken from each block and used for the 
measurements of the biofilm's height and viability. To obtain the three-dimensional structure information, the biofilms were observed under the "Stack" model of the Zen software (Zeiss).

\section{Results and discussion}

\subsection{Microbial reduction of the GO under different growth phases of anodic biofilm}

As shown in Fig. 1a, after the first EPC, some small pieces with irregular shapes were found on the surface of the graphite felt, which was confirmed by FESEM. The magnification of the rough structure, seen in Fig. 1a, was present in Fig. 1b, where we found that the structure was stacked in small irregular pieces and internal caves were formed. At the initial formation stage of the biofilms, small amounts of bacteria were found landing on the surface of the rough structure. For the third and the fifth EPC, the surface morphology of the anode was also examined by FESEM. As shown in Fig. 1c and d, we discovered some much larger rough structures on the graphite felt compared with those found at the first EPC, which contained a large interior of cavities and holes. This rough surface and three-dimension structure apparently provided a larger specific surface area for microbes to settle.

$\mathrm{X}$-ray diffraction was utilized to investigate the rough structure on the anode at the end of the first EPC (1-mrGO anode), the third EPC (3-mrGO anode) and the fifth EPC (5-mrGO anode) by comparison with the GO powder. As shown in Fig. 2a, the diffraction peak of the GO powder appears at an angle of $2 \theta=10.19^{\circ}$, which was near the typical intense crystalline peak of GO of $10.27^{\circ},{ }^{35}$ and the interlayer spacing was of $0.86 \mathrm{~nm}$. The GO powder $\left(1 \mathrm{mg} \mathrm{mL}^{-1}\right)$ was added to the anode chamber with the anodic medium and glucose. After reduction by the anodic bacteria, approximately 90 hours in the first EPC, X-ray diffraction of 1 -mrGO showed that $10.19^{\circ}$ of the peak disappeared and a sharp peak appeared at $2 \theta=24.80^{\circ}$, whereby the interlayer spacing decreased to $0.36 \mathrm{~nm}$. The dramatic drop in the interlayer spacing between the GO and 1-mrGO demonstrated the removal of oxygen and water from the interlayer and provided clear evidence of the reduction. Fig. 2a also showed that similar sharp peaks were obtained for 3-mrGO $\left(2 \theta=10.25^{\circ}\right)$ and 5 -mrGO $\left(2 \theta=10.28^{\circ}\right)$, which further confirmed the significant GO reduction during the long running process of the MFC. ${ }^{35}$

Furthermore, we characterized the reduction of the GO flakes using Raman spectroscopy. Changes in Raman band intensity and shifts provide information on the nature of $\mathrm{C}=\mathrm{C}$ double bonds and defects. Fig. 2b shows four Raman spectral curves of the GO before and after the reduction by the anodic microbes for the GO, 1-mrGO, 3-mrGO and 5-mrGO. The GO powder was tested before it was added to the anode chamber, and two peaks were obtained in the Raman spectroscopy: $1359 \mathrm{~cm}^{-1}$ for the $\mathrm{D}$ band and $1605 \mathrm{~cm}^{-1}$ for the $\mathrm{G}$ band, which were very similar to those reported by a previous study showing microbial GO reduction. ${ }^{35,36}$ In the Raman spectra of the mrGO

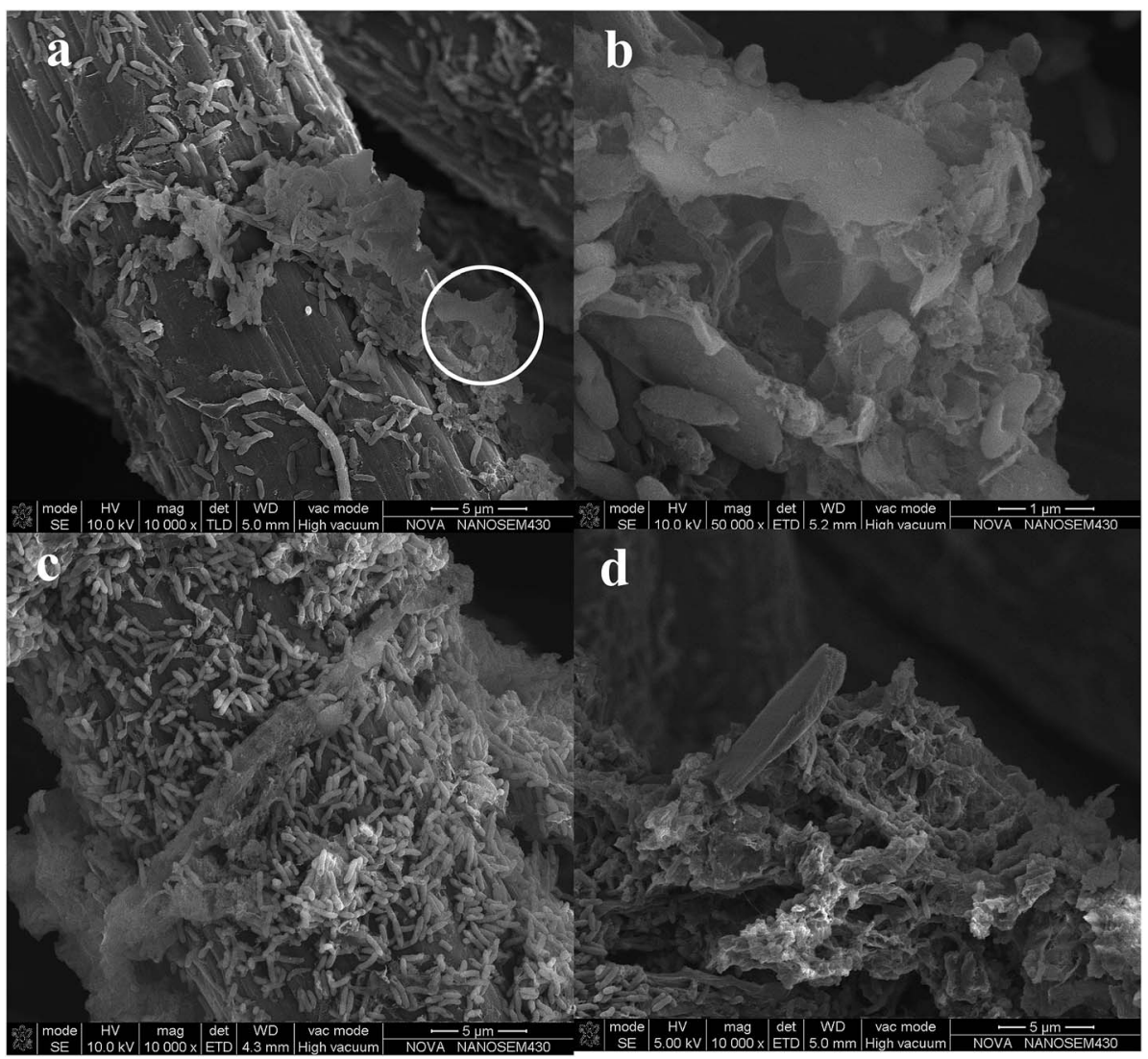

Fig. 1 FESEM of morphology for anode of 1-MFC (a and b), 3-MFC (c), 5-MFC (d). 

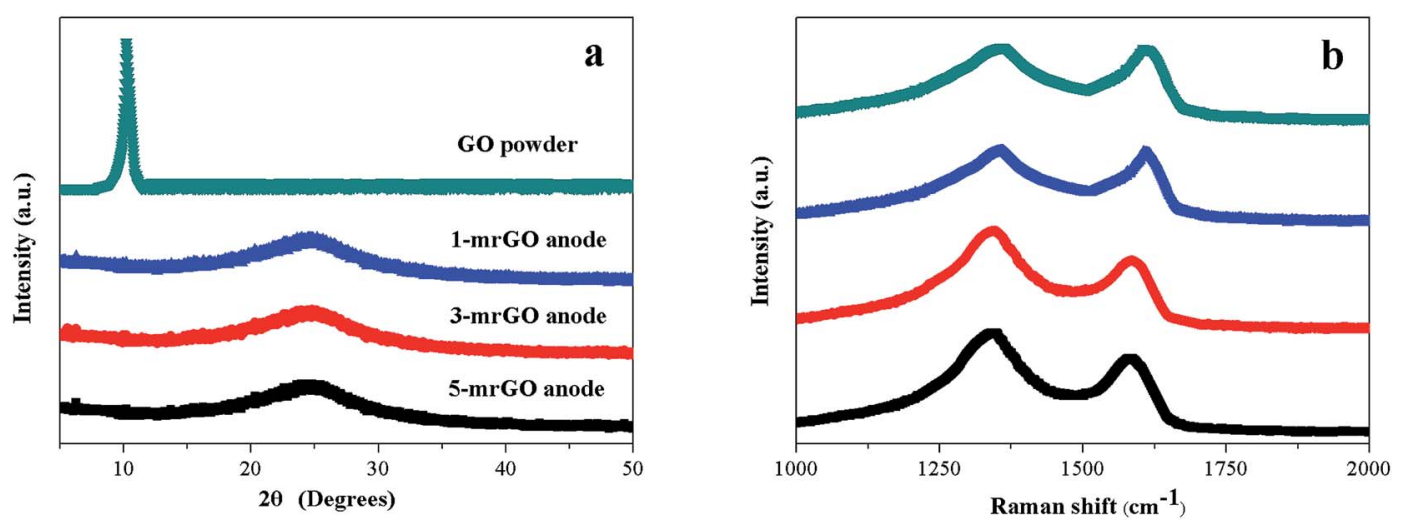

Fig. 2 X-ray diffraction (a) and Raman spectra (b) of anodes for different MFCs.

(Fig. 2b), the D band was shifted to $1350 \mathrm{~cm}^{-1}, 1344 \mathrm{~cm}^{-1}$ and $1342 \mathrm{~cm}^{-1}$, and the $\mathrm{G}$ band was shifted to $1587 \mathrm{~cm}^{-1}, 1585 \mathrm{~cm}^{-1}$ and $1582 \mathrm{~cm}^{-1}$ for 1 -mrGO, 3-mrG and 5-mrGO, respectively. With the increased contact time between the mrGO and the microbes, the left shift of the D band and the $\mathrm{G}$ band indicated that the GO was reduced. ${ }^{37}$ The intensity ratio of the $\mathrm{D}$ band to the $\mathrm{G}$ band $\left(I_{\mathrm{D}} / I_{\mathrm{G}}\right)$ increased steadily in the process of reduction, of which the GO powder was 1.00 , the $1-\mathrm{mrGO}$ was 1.06 , the 3-mrGO was 1.11 and the 5 -mrGO was 1.14 . This pattern of results was in good agreement with the previous studies, strongly confirming the GO reduction..$^{35,36}$

\subsection{Biofilm evolution and viability during GO reduction}

The BCA protein assay was used to characterize the microbial biomass on the anode to evaluate the influence of the mrGO on formation of the anodic biofilms. As shown in Fig. 3, the total protein of the anodic biofilm on different MFCs was tested in the first EPC, the fourth EPC and the fifth EPC. The results clearly showed after the first EPC that for MFC-B $\left(11.52 \mu \mathrm{g} \mathrm{m}^{-2}\right)$ and MFC-C $\left(11.78 \mu \mathrm{g} \mathrm{m}^{-2}\right)$, the anodes acquired less total biofilm protein than that of the mrGO-free MFC-A $\left(21.84 \mu \mathrm{g} \mathrm{m}^{-2}\right)$, which revealed the immediate antibacterial activity of GO

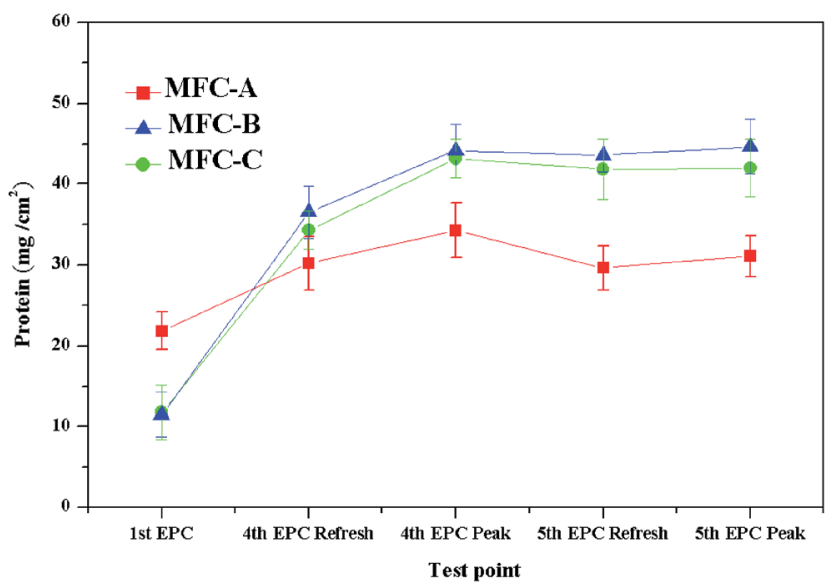

Fig. 3 Total protein curve of anodic biofilm for different MFCs at different test points. toward anodic microbes. J. Chen reported that the wrinkled and crumpled three-dimensional structure of the graphenemodified anode could largely increase the height of biofilm thickness on the anode in a short time, which suggested that the three-dimensional structure of the mrGO had not been completely built in the first EPC. After three EPCs, the total protein of MFC-B and MFC-C both substantially increased to $36.54 \mu \mathrm{g} \mathrm{m}^{-2}$ and $34.31 \mu \mathrm{g} \mathrm{m}^{-2}$, respectively, while that of the MFC-A increased to $30.23 \mu \mathrm{g} \mathrm{m}^{-2}$. The larger increase of the total protein in mrGO-modified anodes demonstrated that more bacteria were attached to the surface of anode with the three-dimensional mrGO structure after three EPCs. When the voltage of the three different MFCs increased to the maximum in the fourth EPC, all the MFCs exhibited a considerable increase in the total protein since the bacteria on the anode reproduced very quickly during this period. After the medium was changed, the total protein on the anodic biofilm slightly decreased in all three MFCs. The rates of decrease for the MFC$B$ and MFC-C were $1.29 \%$ and $3.10 \%$, respectively, which were much lower than those of the MFC-A (13.55\%). Due to the formation of the mrGO on the anode, which had a threedimensional structure and crumpled surface, the acquired bacteria firmly adhered to the anode and were less detached from the anode in long term MFC operation. The total protein was also tested at the peak of output voltage in the fifth EPC for all the three MFCs. The results showed that the MFC-A $\left(31.11 \mu \mathrm{g} \mathrm{m}^{-2}\right)$ was $9.32 \%$ lower than that tested at the peak of the fourth EPC, while the statistics of MFC-B $(-1.25 \%)$ and MFC-C $(2.7 \%)$ were more stable. The variation in the total protein between the two neighboring voltage peaks revealed that microbial adhesion recovered more slowly after the anodic medium refreshed for the mrGO-free anode, while the other two mrGO-modified anodes dynamically recovered well. This also implied that the latter two had already reached a steady state in the formation of biofilms.

Apart from the total protein to quantify the anodic microbial amount in different stages of the operation, the thickness and viability of the biofilms were also further examined by CSLM (Fig. 4a and b) to clarify other biofilm effects aroused by the formation of the mrGO. All of the biofilms were tested by CSLM from the top of the electrode to the surface. As reported by 

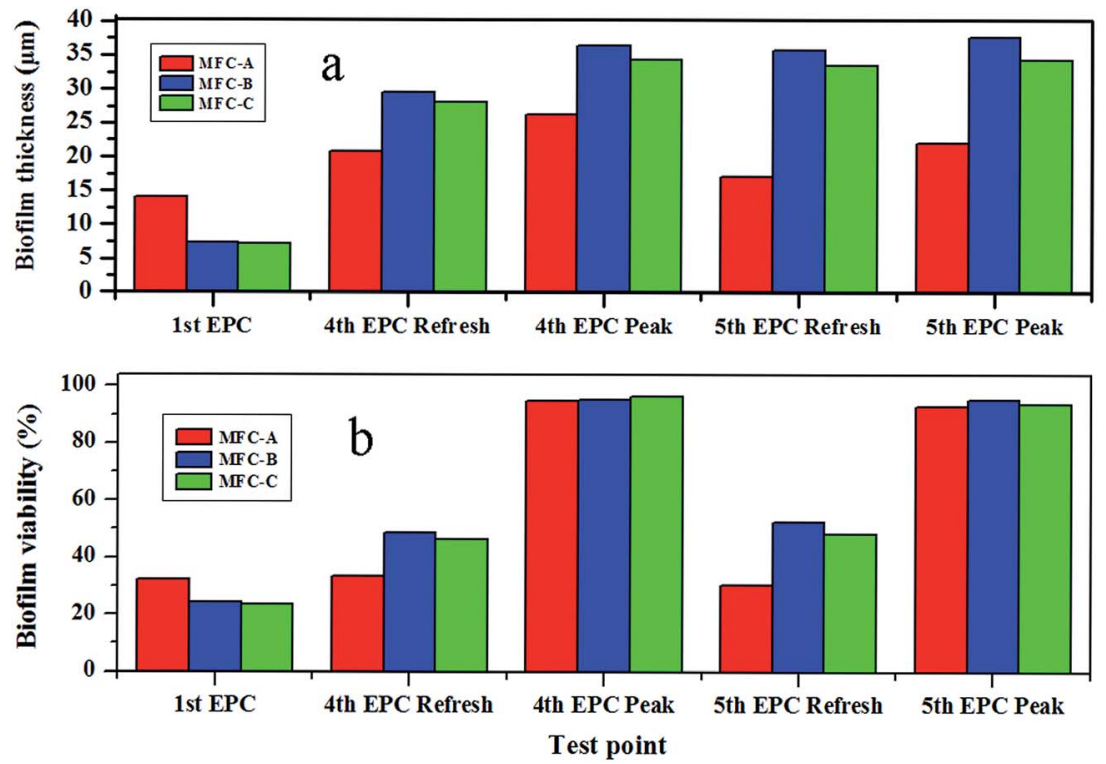

Fig. 4 Biofilm thickness (a) and viability (b) of anodic biofilm for different MFCs at different test points.

Suzanne,,$^{38}$ the cross section of $20 \mu \mathrm{m}$ from the top of the electrode was set as a comparative point to evaluate the viability of the whole biofilm. Similar to the results of the total protein test, after the first EPC, the GO-free anode of MFC-A acquired the highest biofilm thickness $(14.02 \mu \mathrm{m})$, which was far more than that of the other two GO-added MFCs $(7.23 \mu \mathrm{m}$ for MFC-B and $7.14 \mu \mathrm{m}$ for MFC-C). The biofilm viability test, for all the anodes, was in accordance with the result of the biofilm thickness test after the first EPC, of which, the GO-free anode had the highest biofilm viability $(32.31 \%)$. This was a little better than the other two (24.32\% for MFC-B and $23.58 \%$ for MFC-C). Since the in situ formation of the mrGO on the anode was incomplete, and biofilm was still immature after the first EPC, the anodes with the addition of the GO dispersion did not gain any advantage in both biofilm thickness and viability. Instead, they were mildly influenced by the high concentration of GO dispersion in the formation of the biofilms. As shown in Fig. 4, by the beginning of the fourth EPC, the biofilm thickness of the anodes all increased, with MFC-B and MFC-C increasing to $29.44 \mu \mathrm{m}$ and $28.02 \mu \mathrm{m}$, respectively, which were much higher than that of the GO-free MFC-A $(20.73 \mu \mathrm{m})$. The biofilm viability was also largely improved for MFC-B (48.46\%) and MFC-C (46.16\%), but the growth for MFC-A (33.22\%) was much less. The greater promotion in the thickness and viability on the anodic biofilms of MFC-B and MFC-C, compared to MFC-A, clearly came from the complete formation of the mrGO on the anode (as shown in Fig. 1). As the voltage of all the MFCs reached the peak in the fourth EPC, the thickness of biofilm increased to $36.52 \mu \mathrm{m}$ and $34.34 \mu \mathrm{m}$ for MFC-B and MFC-C, respectively. These increases were far more than that of the GO-free MFC-A $(26.21 \mu \mathrm{m})$. Meanwhile, the biofilm viability of all the anodes increased to between $94.00 \%$ and $97.00 \%$. The small change in statistics for thickness in the GO-added MFCs in this period demonstrated that the anodic biofilms tended to be mature and stable. After changing the medium at the beginning of the fifth EPC, the thickness on anodes of MFC-A dramatically dropped over 34.5\% to $17.17 \mu \mathrm{m}$, owing to the hydraulic actions and natural falling off. However, the GO-added anode thickness changed little, with the MFC-B decreasing by $1.72 \%$ to $35.89 \mu \mathrm{m}$ and the MFC$\mathrm{C}$ which decreasing by $2.39 \%$ to $33.52 \mu \mathrm{m}$. The variation in the rate of the biofilm thickness in this period provided a further argument that the anodic biofilm was matured for these two MFCs. At the beginning of the fifth EPC, anodic microbes were in the initial stage of growth, and a sharp drop in the viability of all the anodic biofilms was recorded. When the output voltage peaked in the fifth EPC, the thickness of the anodes for MFC-B and MFC-C recovered to $37.71 \mu \mathrm{m}$ and $34.33 \mu \mathrm{m}$, respectively, which were very close to the data recorded at the voltage peak of the last EPC. However, the biofilm of the GO-free anode did not recover well, since its thickness $(22.11 \mu \mathrm{m})$ was reduced by $15.6 \%$ compared to that tested at the peak of voltage in the last EPC. This difference in recovery of the biofilm height agreed with the total protein mentioned above, and it also derived from the high specific area of the mrGO and the strong adhesion between the mrGO and bacteria. In this period, the biofilm viability of the anodes all reached $92.00-95.00 \%$.

The TEM of the anodic solution was also tested after medium replacement and the addition of the GO dispersion, which was used to characterize the contact of the mrGO/GO flakes and the bacteria suspended in the anodic solution. The graph of TEM (Fig. 5) revealed that some microbes began to land on the surface of the mrGO/GO flakes before it was attached to the electrode, and the reducing reaction of the GO began in this period. It also indicated that biofilm viability of the GO-added MFCs did not perform well at the beginning of the first EPC, partly due to the physical contact between the bacteria and the mrGO/GO flakes in advance. This conclusion agreed well with the statistics from CSLM. As we know from the viability results shown in Fig. $4 \mathrm{~b}$, each time after we added the GO dispersion at the beginning of the EPC, for the MFC-C, the 

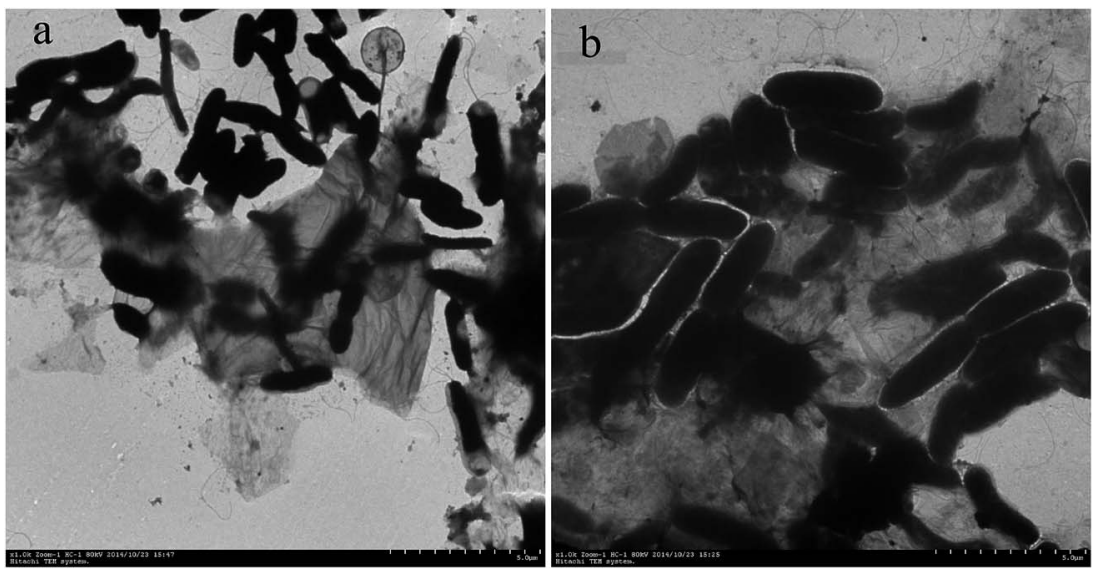

Fig. 5 TEM of mrGO flakes suspended in anodic solution.

viability was apparently affected compared to that of MFC-B. The statistical gap between MFC-B and MFC-C clearly showed that the highly concentrated GO flakes in the anodic solution exerted negative effects on the anodic biofilm formation. The highly concentrated GO dispersion meant more contact between the GO flakes and bacteria exposed to the outside and led to more severe membrane diapirism..$^{31}$ Despite the negative influence on the biofilm viability caused by the mrGO, the process of biofilm formation improved with the mrGO flakes coating on the anode at the same time. The total protein and biofilm thickness were both better than those of the mrGO-free anode each time the biofilm began to recover after the medium was refreshed.

\subsection{Performance improvement of MFC by the graphene/ exoelectrogen composite biofilm electrode}

The voltage output curves for the three types of MFCs are shown in Fig. 6a. In the first EPC, the maximum voltage of the MFC-A $(0.349 \mathrm{~V})$ was clearly higher than those of the MFC-B $(0.258 \mathrm{~V})$ and the MFC-C $(0.262 \mathrm{~V})$. This phenomenon is most likely attributed to two main reasons. On one hand, some small flakes of the mrGO began to contact the microbes at the initial stage of the biofilm formation, and the antibacterial activity of graphene was triggered. As a previous paper reported, direct physical contact between graphene and bacteria could weaken the viability of the bacteria, leading to a decrease in the electrochemical activity. ${ }^{39}$ On the other hand, the electrons transferred from the microbes are partly utilized to reduce the GO after addition of the GO dispersion in the MFC-B and the MFC-C. The majority of electrons should be transferred to the surface of the anode by the electron transfer chain under normal conditions without the GO. ${ }^{36}$ In the second set of EPCs, a huge change in the voltage gaps are shown in Fig. 6, where the voltage output of the two the GO-added MFCs increased significantly in this period, and both were higher than that of the GO-free MFC-A. Better performance in the voltage output of these two MFCs showed that the negative factors resulting from the GO dispersion waned in the second set of EPCs. As shown in Fig. 6a, the voltage outputs of the MFCs stabilized after the first two
EPCs since the shape of the graphs became very similar and the maximum of voltage output fluctuated lower. For voltage output of MFC-A, it always took more than 20 hours to achieve the maximum value in the latter four EPCs, while the other two could reach that level in 10 hours, which provided evidence that the mrGO-modified anode could more easily lead to a stable level of electricity production. In Fig. 6, we also determined the fluctuation of the MFC-A in the fifth EPCs, of which the GO-free MFC-A acquired a rather low maximum voltage output value, and the change rate was as high as $8.12 \%$. In contrast to this uncontrolled change, the fluctuation rates in the maximum voltage values for the GO-added MFCs were all below $\pm 2.20 \%$ in the latter four EPCs. In the fourth and fifth EPCs, the voltage output apparently agreed with the statistics in terms of viability and height of the biofilms for all three MFCs, which provided further evidence that the mrGO-modified anode could recover the biofilms well and maintain stable electricity production. For the two GO-added MFCs, MFC-B obviously acquired a higher performance in voltage output than MFC-C, suggesting that maintenance of a high concentration of GO dispersion in every EPC would have a negative impact on electricity production process, especially on the viability of the biofilms which was mentioned above.

Apart from the voltage output, power density and polarization were also used to evaluate the electricity production performance of the MFCs. As shown in Fig. 6b, in the first EPC, the power density of the MFCs was highly consistent with the statistics of voltage output. The MFC-A achieved the highest maximum power density value of $390.45 \mathrm{~mW} \mathrm{~m}^{-2}$, which was $38.17 \%$ and $43.74 \%$ higher than that of MFC-B $\left(282.59 \mathrm{~mW} \mathrm{~m}^{-2}\right)$ and MFC-C (271.63 $\left.\mathrm{mW} \mathrm{m}^{-2}\right)$, respectively. In the fifth EPC, the MFC-A power density reached a peak of $690.45 \mathrm{~mW} \mathrm{~m}^{-2}$, which was much lower than those of MFC-B $\left(998.56 \mathrm{~mW} \mathrm{~m}^{-2}\right)$ and MFC-C (1140.63 $\mathrm{mW} \mathrm{m}^{-2}$ ) owing to the less bacterial quantity of biofilm and the lack of the advantage in electricity transfer brought by the mrGO. For MFC-B and MFC-C, the difference in the power density was not large and the slopes of curves were very close, but the defect resulted from the high concentration of the GO flakes in every EPC to prevent the MFC-C from reaching the maximum value, as high as that of MFC-B. 

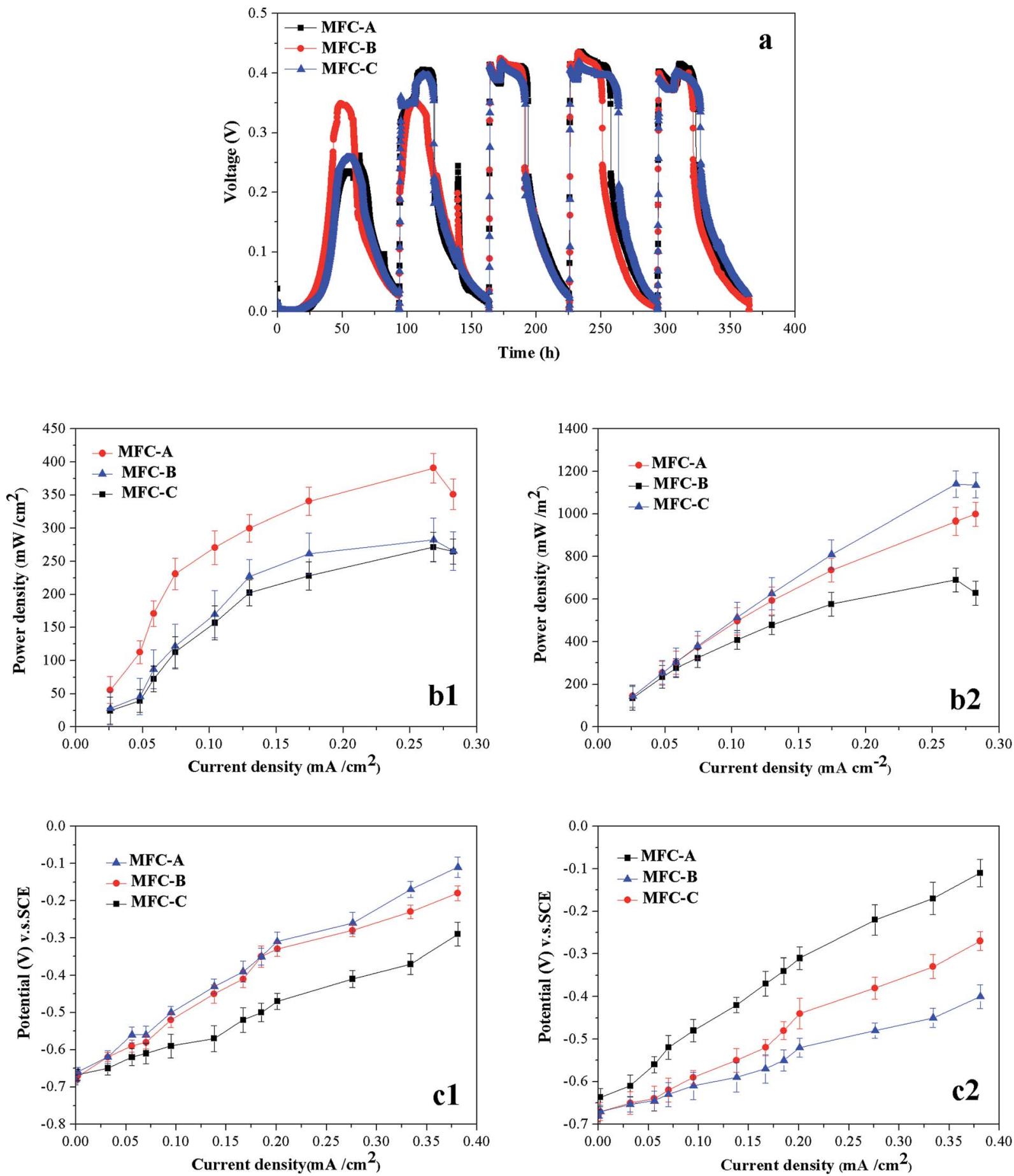

Fig. 6 Voltage output of different MFCs in five EPCs (a), and power density curves (b) and polarization curves (c) of different MFCs in the first EPC and fifth EPC.

A polarization curve was used to measure the difficulty of the electrochemical reaction being derived. As shown in Fig. 6c, in the first EPC, the voltage of open circuit of the three MFCs was similar. As the current increased from 0 to $0.381 \mathrm{~mA} \mathrm{~cm}^{-2}$, the anode potential of MFC-A changed from $-0.68 \mathrm{~V}$ to $-0.29 \mathrm{~V}$, and the slope of the curve was very near that of the other two MFCs. The GO-free MFC-A obtained higher absolute values of the potential voltage as we provided a value of the current intensity, which indicated that for the MFC-A, the over potential of electrochemical reaction was lower and the reaction rate was faster on the surface of anode. The figure also showed that, in the fifth EPC, the GO-added MFCs acquired higher absolute values of potential voltage than the GO-free MFC-A at the same value of current intensity. In this EPC, the voltage of the open circuit for the MFC-B was very close to that for the MFC-C. However, the curve of the MFC-B had a higher slope, which 
demonstrated that the addition of the GO dispersion only in the first electricity production cycle could lead to a lower overpotential and a faster electrochemical reaction on anode, rather than the GO dispersion addition in every electricity production cycle.

\section{Conclusions}

The biofilm formation was negatively affected in the first electricity production cycle in terms of viability and bacterial amounts due to antibacterial activity of GO at high concentration. However, the mrGO/exoelectrogen composite biofilms were formed well after the three electricity production cycles, which overcame the antibacterial activity of GO/mrGO, as the result of enhanced bacterial adhesion and accelerated biofilm recovery in the presence of mrGO due to its high specific area for bacteria to settle and high conductivity for enhanced electrons transfer between bacteria and anode.

\section{Conflicts of interest}

There are no conflicts to declare.

\section{Acknowledgements}

The authors gratefully thank the financial support provided by the National Natural Science Fund of China (No. 21477039, No. 51108186), Scientific and Technological Planning Project of Guangzhou, China (201607010318), Natural Science Fund of Guangdong province, China (No. 2016A030313693), and Guangdong Special Support Program for Training High Level Talents, China (2015TQ01Z039), Science and Technology Planning Project of Guangdong province, China (2014A020216042).

\section{References}

1 B. Logan, B. Hamelers, R. Rozendal, U. Schroder, J. Keller, S. Freguia, P. Aelterman, W. Verstraete and K. Rabaey, Environ. Sci. Technol., 2009, 40, 5181.

2 F. Zhao, R. Slade and J. Varcoe, Chem. Soc. Rev., 2009, 38, 1926.

3 A. Franks and K. Nevin, Energies, 2010, 3, 899.

4 U. Schroder, Phys. Chem. Chem. Phys., 2007, 9, 2619.

5 S. Cheng and B. Logan, Electrochem. Commun., 2007, 9, 492.

6 A. Peter, F. Stefano and K. Jurg, Appl. Microbiol. Biotechnol., 2008, 78, 409.

7 P. Avouris, Z. Chen and V. Perebeinos, Nat. Nanotechnol., 2007, 2, 605.

8 K. Novoselov, A. Geim, S. Morozov, D. Jiang, Y. Zhang, S. Dubonos, I. Grigorieva and A. Firsov, Science, 2004, 306, 666.

9 A. Balandin, S. Ghosh, W. Bao, I. Calizo, D. Teweldebrhan, F. Miao and C. Lau, Nano Lett., 2008, 8, 902.

10 J. Xia, F. Chen, J. Li and N. Tao, Nat. Nanotechnol., 2009, 4, 505.

11 S. Wang, P. Ang, Z. Wang, A. Tang, J. Thong and K. Loh, Nano Lett., 2009, 10, 92.
12 A. Geim and K. Novoselov, Nat. Mater., 2007, 6, 183.

13 A. Castro Neto, F. Gunea, N. Peres, K. Novoselov and A. Grim, Rev. Mod. Phys., 2009, 81, 109.

14 X. Wang, L. Zhi and K. Mullen, Nano Lett., 2007, 8, 323.

15 Z. Wu, W. Ren, D. Wang, F. Li, B. Liu and H. Cheng, ACS Nano, 2010, 4, 5835.

16 C. Lu, H. Yang, C. Zhu, X. Chen and G. Chen, Angew. Chem., Int. Ed., 2009, 48, 4785.

17 Y. Zhu, S. Murali, W. Cai, X. Li, J. Suk, J. Potts and R. Ruoff, Adv. Mater., 2010, 22, 3906.

18 L. Qu, Y. Liu, J. Baek and L. Dai, ACS Nano, 2010, 4, 1321.

19 J. Roy-Mayew, D. Bozym, C. Punckt and I. Aksay, ACS Nano, 2010, 4, 6203.

20 G. Huang, T. Chen, Z. Wang, K. Chang and W. Chen, J. Power Sources, 2013, 235, 122.

21 D. Benson, Y. Li, W. Luo, R. Ahuja, G. Svenssion and U. Haussermann, Inorg. Chem., 2013, 52, 6402.

22 Y. Zhang, G. Mo, X. Li, W. Zhang, J. Zhang, J. Ye, X. Huang and C. Yu, J. Power Sources, 2011, 196, 5402.

23 J. Liu, Y. Qiao, C. Guo, S. Lim, H. Song and C. Li, Bioresour. Technol., 2012, 114, 275.

24 M. McAllister, J. Li, D. Adamson, H. Schniepp, A. Abdala, J. Liu, M. Herrera-Alonso, D. Milius, R. Car, R. Prud'homme and I. Aksay, Chem. Mater., 2007, 19, 4396.

25 H. Schniepp, J. Li, M. McAllister, H. Sai, M. Herrera-Alonso, D. Adamson, R. Prud'homme, R. Car, D. Saville and A. Aksay, J. Phys. Chem. B, 2006, 110, 8535.

26 Y. Yong, X. Dong, M. Chan-Park, H. Song and P. Chen, ACS Nano, 2012, 6, 2394.

27 O. Akhavan and E. Ghaderi, Carbon, 2012, 50, 1853.

28 Y. Huang, X. Liu, J. Xie, G. Sheng, G. Wang, Y. Zhang, A. Xu and H. Yu, Chem. Commun., 2011, 47, 5795.

29 Y. Yuan, S. Zhou, B. Zhao, L. Zhuang and Y. Wang, Bioresour. Technol., 2012, 116, 453.

30 L. Zhuang, Y. Yuan, G. Yang and S. Zhou, Electrochem. Commun., 2012, 21, 69-72.

31 S. Liu, T. Zeng, M. Hofmann, E. Burcombe, J. Wei, R. Jiang, J. Kong and Y. Chen, ACS Nano, 2011, 5, 6971.

32 S. Stankovich, R. Piner, X. Chen, N. Wu, S. Nguyen and R. Ruoff, J. Mater. Chem., 2006, 16, 155.

33 D. Dikin, S. Stankovic, E. Zimney, R. Piner, G. Dommett, G. Evmenenko, S. Nguyen and R. Ruoff, Nature, 2007, 448, 457.

34 D. Lovley and E. Phillips, Appl. Environ. Microbiol., 1998, 54, 1472.

35 S. Gurunathan, J. Han, V. Eppakayala and J. Kim, Colloids Surf., B, 2013, 102, 772.

36 G. Wang, F. Qian, C. Saltikov, Y. Jiao and Y. Li, Nano Res., 2011, 4, 563.

37 Z. He and Y. Zhu, Mater. Sci. Semicond. Process., 2014, 27, 1013.

38 F. Suzanne, L. Alicia, S. Faustino and M. José, Adv. Appl. Microbiol., 2010, 71, 113.

39 J. Chen, D. Feng, Y. Hu, J. Sun and Y. Yang, J. Power Sources, 2015, 290, 80. 